\title{
Application of Magnetic Materials in Inductive Sensors for Partial Discharge Activity Monitoring
}

\author{
J. Petrášš, J. Džmura, J. Kurimský, R. Cimbala, I. Kolcunová, \\ B. DOLnÍK And J. ZBOJOVSKÝ \\ Technical University of Košice, Letná 9, 04200 Košice, Slovakia
}

\begin{abstract}
The measurement and monitoring of partial discharge activity is one of the commonly used methods to determine the status of insulation system in high voltage power devices. For such monitoring there are various methods involving direct galvanic coupled measurement, acoustic method, or measurement by inductive method. Inductive measurement of partial discharges uses inductive sensors, which sense the partial discharge pulses occurring in high voltage circuit. The sensitivity of such sensors depends on their design, construction, and materials used for sensor core. The choice of magnetic material is very important to achieve optimal values of sensor sensitivity. We have constructed experimental sensors using different magnetic materials and different construction. In our experiments we have selected two magnetic material types: magnetic material based on $\mathrm{Fe}-\mathrm{Ni}$ (permalloy) and magnetic material based on $\mathrm{MO} \cdot \mathrm{Fe}_{2} \mathrm{O}_{3}$. We were able to examine the optimal construction type and magnetic material of sensor core for these sensors. The minimum acceptable sensitivity of the sensors was determined by equivalent measuring sensitivity when using direct galvanic method.
\end{abstract}

DOI: 10.12693/APhysPolA.137.714

PACS/topics: partial discharge, inductive sensors

\section{Introduction}

Long lifetime and reliability are requirements that have to be met for electric device insulation system and other materials required for high voltage electric power device area of industry.

High voltage devices used to have complicated shape, construction, and electrode system configuration. There are various types of faults causing partial discharges, e.g., in stator insulation [1]. This is even more complicated when ionizing and non-homogeneous electric fields are applied. Partial discharge activity is one of the key quantitative indicators of the status and health of high voltage electric insulation system. For partial discharge activity monitoring there are various methods including measurements using inductive sensors [2]. The sensitivity of such sensors strongly depends on their construction and materials used [3]. The choice of magnetic material is very important to achieve optimal values of sensor sensitivity [4].

\section{Features of inductive sensors}

We have proposed a sensor design starting from the fact that it is associated with the circuit of the measured device [5]. Figure 1 shows the layout and the construction diagram of the sensor. The reciprocal coefficient of the induction can be calculated using

$$
M_{12}=\frac{\mu_{0} \mu_{r}}{2 \pi} N_{2} h \ln \left(\frac{r_{2}}{r_{1}}\right),
$$

and the self-inductivity by using

*corresponding author; e-mail: jaroslav.petras@tuke.sk

$$
L_{2}=\frac{\mu_{0} \mu_{r}}{2 \pi} N_{2}^{2} h \ln \left(\frac{r_{2}}{r_{1}}\right) .
$$

In case of the transformation coefficient and the magnetic constant, they are equal to $k=M_{12} / L_{2}=1 / N_{2}$, and $\mu_{0}=1.253 \times 10^{-6} \mathrm{H} / \mathrm{m}$, respectively. Next, $\mu_{r}$ is relative permeability of the toroid material, $N_{2}$ is the number of secondary coil windings, $h$ is the height of the toroid, while $r_{1}$ and $r_{2}$ are inner and outer radii of the toroid. The special feature of this construction of the sensor is the independence of the transformation coefficient on the form of the primary wire. The transmission function can be calculated using

$$
\frac{u_{2}}{i_{1}}=\frac{M_{12} R}{L_{2}} \frac{1}{\sqrt{1+\left(\frac{R}{\omega L}\right)^{2}}}=\frac{R}{N_{2}} \frac{1}{\sqrt{1+\left(\frac{R}{\omega L_{2}}\right)^{2}}} .
$$

As one can see, this function depends on the frequency and it means also that the inductivity of the circuit changes according to the permeability of the core material. Furthermore, the value of $\mu_{r}$ depends also on the frequency. From the above-mentioned equations, we can state that the conditions are fulfilled for small alternating or pulse currents. The input of the secondary coil behaves as an electric circuit with high input impedance. Lower number of windings ensures higher resistance to the external noise signals.

For operation in the area of alternating currents the non-retentive materials with coercive force $H_{e}>800 \mathrm{~A} / \mathrm{m}$ are suitable. They have thin hysteresis curve and high values of initial permeability $\mu_{p}$ and maximal permeability $\mu_{m}$. Furthermore, the advantage of the designed inductive sensor is that it can measure also the leakage current of the insulation system at the frequency of $50 \mathrm{~Hz}$, which is another parameter indicating the status of the insulation material [6]. 

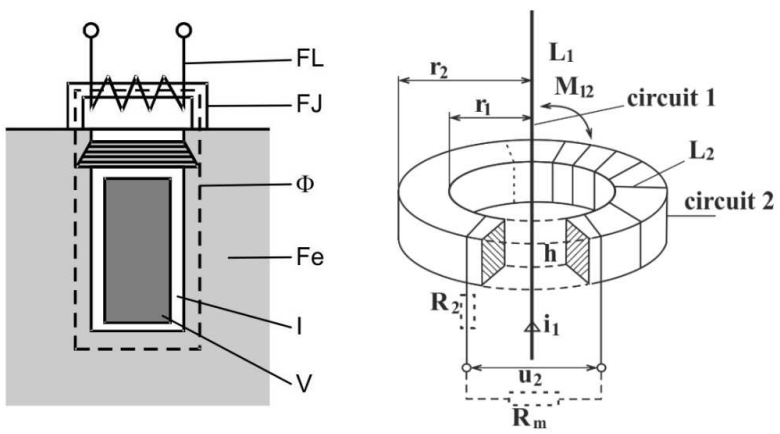

Fig. 1. Inductively coupled sensor - the situation and sensor detailed view [7], where FL - sensor winding, FJ - sensor, $\Phi$ - magnetic flow, Fe - stator steel plate, $\mathrm{I}$ - insulation, $\mathrm{V}-\mathrm{Cu}$ wire.

During the measurement of partial discharge signal by inductive sensors we suppose that the material of the core is not oversaturated, i.e., it operates at very low values of $H$. The initial permeability has significant influence on the measurement sensitivity and this value depends on temperature and frequency. As measured in experiments, $\mu_{r}$ of tested materials changes significantly above frequency of $5 \times 10^{5} \mathrm{~Hz}$.

\section{The model of winding}

According to the configuration of generator pole in stator drain after some technological adaptations the situation of inductively coupled sensor is shown in Fig. 1.

\section{Experimental setup}

For our experiments, we used the inductive method of partial discharge monitoring together with the direct galvanic method for reference. The measurement setup can be seen in Fig. 2.

Our tested sample was a high voltage coil of stator winding. Measurements were done in laboratory conditions in accordance to IEC60270 standard with earthed tested object.

In the first case we used direct measurement method which is reliable and widely used for basic parameter measurement in partial discharge measurement. Tested coil was placed on insulated pole to avoid coil surface touching other objects, thus causing parasite partial discharges. To avoid corona discharges we placed a homogenization electrode to the winding end with high voltage supplied. The drain part of the coil was earthed.

Tested voltage was set to initial value and then increased until initiation voltage is reached for first partial discharges to occur. At this voltage partial discharge parameters were stored. Further, the voltage was increased by steps of $500 \mathrm{~V}$ until nominal voltage of $5 \mathrm{kV}$ was reached. At each voltage level partial discharge parameters were measured and stored repeatedly.

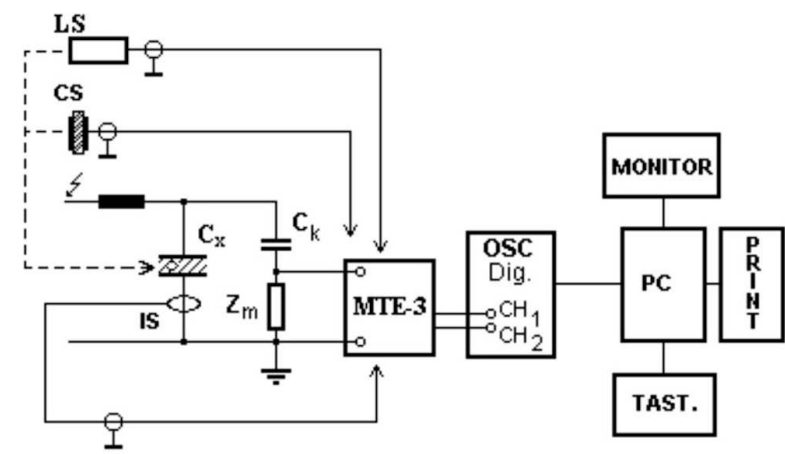

Fig. 2. Electric diagram of the experimental setup [8]. The parts are marked as follows: LS - linear sensor, CS - capacitive sensor, IS - inductive sensor, $C_{x}$ tested object, $C_{k}$ - coupling capacity, MTE-3 - partial discharge measurement device.

For the second set of measurements, indirect measurement method was used with inductive sensor placed in magnetically shielded cover and connected in operating earthing circuit of the high voltage coil. Test voltage was increased until initiation voltage of $2.5 \mathrm{kV}$ was reached. At this level discharge activity was measured and stored. Later the voltage was increased with step of $500 \mathrm{~V}$ until nominal voltage $6 \mathrm{kV}$ was reached. At each voltage level, discharge activity was measured.

The material of the sensor was magnetic material based on $\mathrm{Fe}-\mathrm{Ni}$ (permalloy) and magnetic material based on $\mathrm{MO} \cdot \mathrm{Fe}_{2} \mathrm{O}_{3}$.

\section{The results}

Acquired data were evaluated by phase analysis with statistical methods used for a result set. By these methods phase distributions of maximal and mean values of apparent charge, discharge rate, and amplitude spectrum were calculated. All of these graphs are helping tools for the expert or expert systems during the process of determination of partial discharge type. Each type of partial discharge occurring at different points of a high voltage electric power device falls into different types in categorization range (e.g., corona, surface discharges, etc.) and it has its own characteristic phase distribution of the above-mentioned electrical parameters.

The results shown in Fig. 3 were achieved by direct galvanic method in comparison to the result shown in Fig. 4 for indirect inductive method. As can be observed, all measured statistical parameters calculated from acquired signal are comparable in certain range, although they are not identical. Thus, the sensitivity of inductive sensors is comparable to the sensitivity of galvanic method, and it is acceptable for monitoring purposes. For our experiment, it is important that the results are comparable from both methods of measurement. This can be seen, e.g., on apparent charge maximum value phase distribution graphs, where the peaks of the graphs occur at the same angle of the tested $\mathrm{AC}$ voltage. 

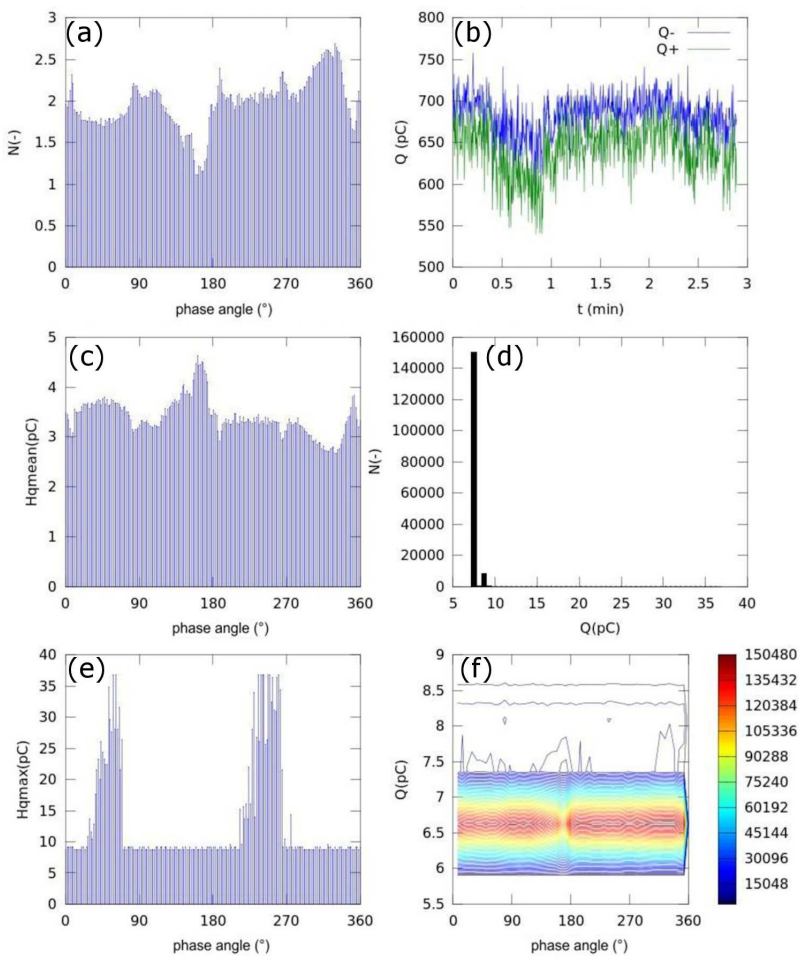

Fig. 3. Phase distribution of partial discharge at nominal voltage $2.5 \mathrm{kV}$, galvanic method.
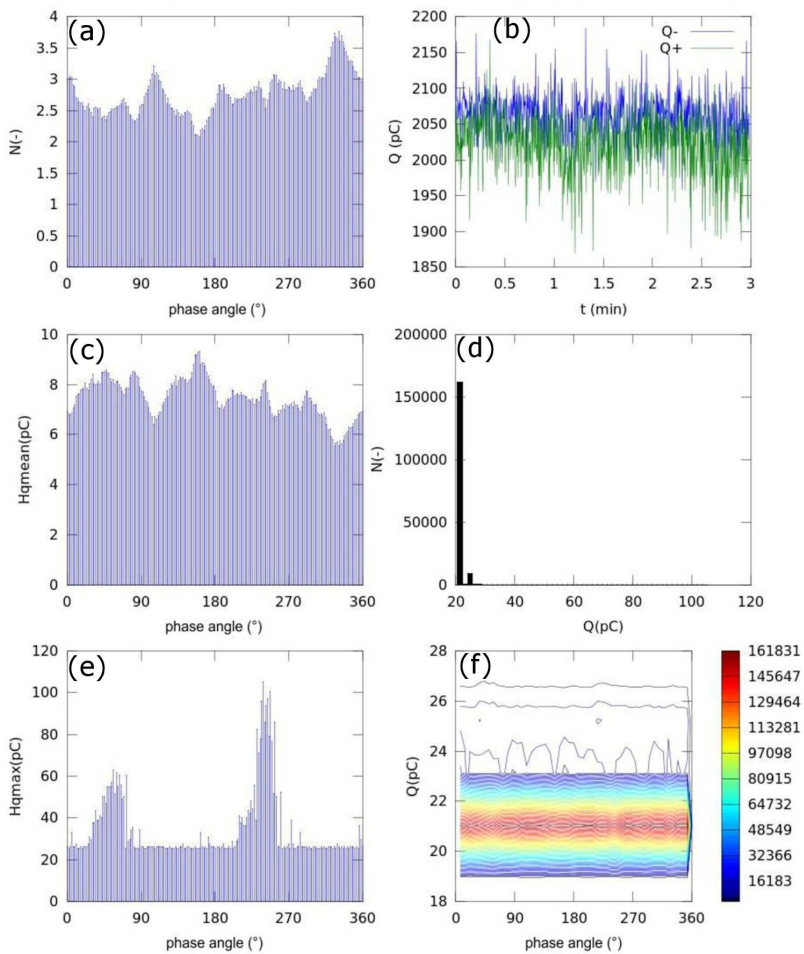

Fig. 4. Phase distribution of partial discharge at nominal voltage $2.5 \mathrm{kV}$, inductive method, coil in stator drain.

\section{Conclusions}

Measurements by direct galvanic and indirect inductive method on coils of electric engine were made for comparison. As we observed, the indirect measurement method using sensors with materials such as magnetic material based on $\mathrm{Fe}-\mathrm{Ni}$ (permalloy) and magnetic material based on $\mathrm{MO} \cdot \mathrm{Fe}_{2} \mathrm{O}_{3}$ provides sufficient sensitivity. This is needed for partial discharge signal acquisition with all important informations in signal retained for statistical evaluation of partial discharge activity. Therefore, we can say that both methods of measurements are equivalent and can be used for partial discharge activity monitoring. Theoretically determined parameters of inductive sensors were verified with our experiments as sufficient for retaining important markers of partial discharge activity type and level in measured signals.

\section{Acknowledgments}

The authors thank Slovak Academy of Sciences and Ministry of Education, projects VEGA No. 1/0340/18 and $2 / 0141 / 16$; Ministry of Education Agency for structural funds of EU projects No. 26220120055; Slovak Research and Development Agency project No. APVV-15-0438.

\section{References}

[1] L. Lisoň, I. Kolcunová, M. Kmec, Acta Electrotechn. Inform. 14, 23 (2014).

[2] J. Balogh, J. Džmura, J. Petráš, Electrotechn. Electron. Automat. 59, 33 (2011).

[3] A. Ali, A. Amir, U. Abdin, M. Zain, IEEE Trans. Instrum. Measur. 68, 4104 (2019).

[4] M. Pavlík, L. Kruželák, M. Mikita, M. Špes, S. Bucko, L. Lisoň, M. Kosterec, L: Beňa, P. Liptai, Archiv. Electr. Eng. 66, 361 (2017).

[5] E. Svenman, A.K. Christiansson, A. Runnemalm, Measurement 146, 396 (2019).

[6] D. Jiao, L.W. Ni, X.L. Zhu, J. Zhe, Z.Y. Zhao, Y.G. Lyu, Z.X. Liu, Sens. Actuat. A 295, 59 (2019).

[7] E. Svenman, Proced. Manuf. 25, 103 (2018).

[8] J. Balogh, J. Petráš, J. Džmura, in: Electrical Engineering and Informatics 6, Proc. Faculty of Electrical Engineering and Informatics of the Technical University of Košice, FEI TU, Košice (Slovakia), 2015, p. 75 . 